\title{
Ultrasonographic optic nerve sheath diameter for predicting elevated intracranial pressure during laparoscopic surgeries: a systematic review and meta-analysis
}

\author{
Eun Jung Kim, MD, PhD • Jae Hee Seo, MD • Bon-Nyeo Koo, MD, PhD • Seung Ho Choi, MD, PhD • Min- \\ Soo Kim, MD, PhD
}

Department of Anesthesiology and Pain Medicine, Anesthesia and Pain Research Institute, Yonsei University College of Medicine, Seoul, Republic of Korea

\section{BACKGROUND}

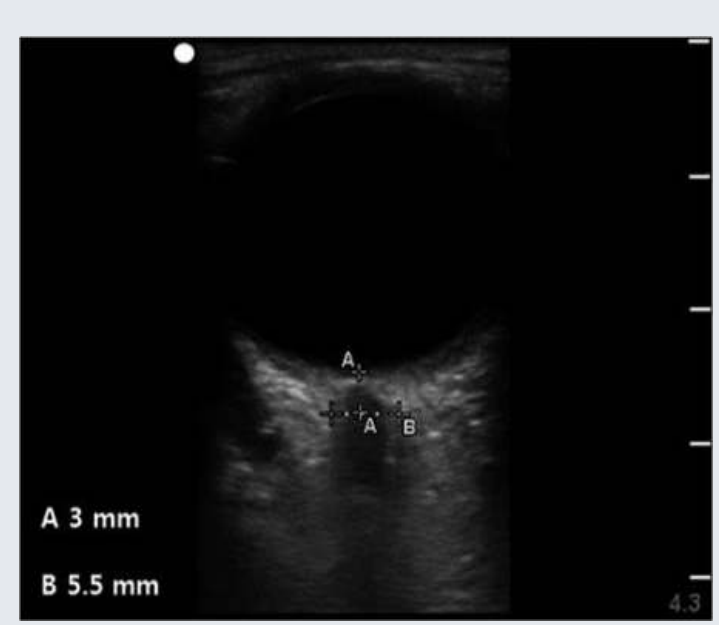

J Endourol. 2014 Jul;28(7):801-6. Increase in intracranial pressure during carbon dioxide pneumoperitoneum with steep trendelenburg positioning proven by ultrasonographic measurement of optic nerve sheath diameter. Kim MS et al
Impact of

pneumoperitoneum on

optic nerve sheath

diameter: not clear due to

inconsistent results from

previous researches

Aim: to investigate the

changes in ONSD during

$\mathrm{CO}_{2}$ pneumoperitoneum as

a predictor for elevated ICP

during laparoscopic surgery

\section{METHODS}

Inclusion: prospective trials in which repeated ONSD measurement was performed to assess the effects of pneumoperitoneum on ICP in patients who had laparoscopic surgery under general anesthesia.

Data search: in August 2016

Primary outcome variables: ONSD changes during the early and late periods, compared with the ONSD values measured after anesthesia induction

\section{RESULTS}

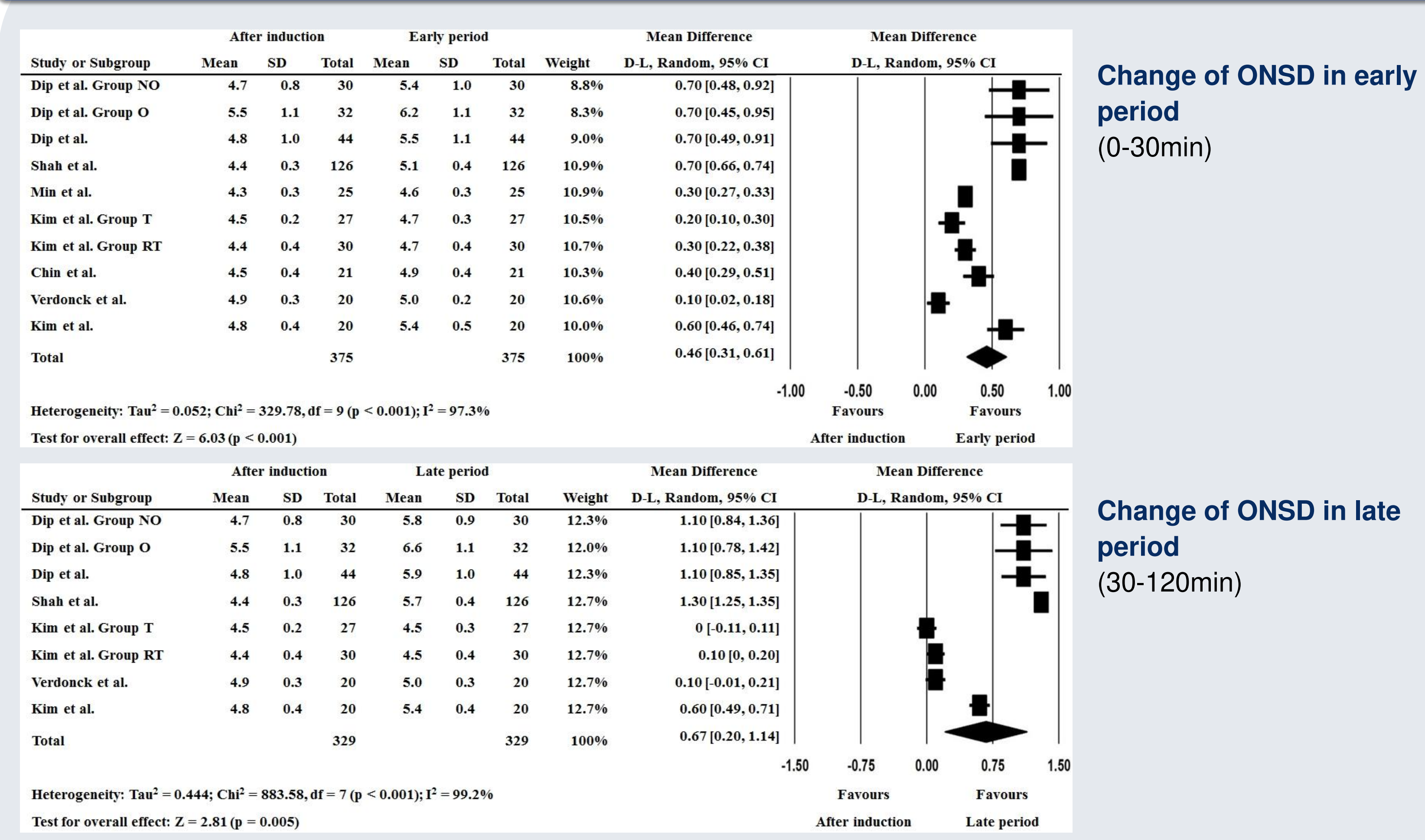

\section{CONCLUSIONS}

Increased ONSD as a predictor for elevated ICP was observed during the early and late periods with $\mathrm{CO}_{2}$ pneumoperitoneum for laparoscopic surgery. 